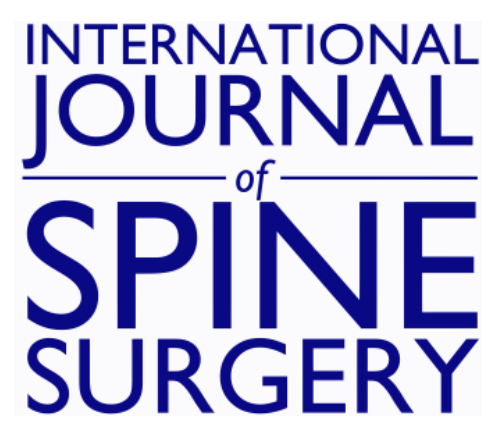

\title{
Deep Surgical-Site Infection Following Thoracolumbar Instrumented Spinal Surgery: The Experience of 25 Years
}

Borja de la Hera, Felisa Sánchez-Mariscal, Alejandro Gómez-Rice, Iria Vázquez-Vecilla, Lorenzo Zúñiga and Esther Ruano-Soriano

Int J Spine Surg 2021, 15 (1) 144-152

doi: https://doi.org/10.14444/8019

http://ijssurgery.com/content/15/1/144

This information is current as of April 26, 2023.

Email Alerts Receive free email-alerts when new articles cite this article. Sign up at: http://ijssurgery.com/alerts 


\title{
Deep Surgical-Site Infection Following Thoracolumbar Instrumented Spinal Surgery: The Experience of 25 Years
}

\author{
BORJA DE LA HERA, MD,${ }^{1,2}$ FELISA SÁNCHEZ-MARISCAL, MD, PHD,${ }^{3}$ ALEJANDRO GÓMEZ-RICE, MD $^{2,5}$ \\ IRIA VÁZQUEZ-VECILLA, MD, ${ }^{1}$ LORENZO ZÚÑIGA, MD,${ }^{1}$ ESTHER RUANO-SORIANO, MD ${ }^{4}$ \\ ${ }^{1}$ Department of Orthopedic Surgery, Getafe University Hospital, Madrid, Spain, ${ }^{2}$ Complutense University, Madrid, Spain, ${ }^{3}$ Department of Orthopedic Surgery, \\ Rey Juan Carlos University Hospital, Madrid, Spain, ${ }^{4}$ Infectious Diseases Unit, Department of Internal Medicine, Getafe University Hospital, Madrid, Spain, \\ ${ }^{5}$ Department or Orthopedic Surgery, Ramón y Cajal University Hospital, Madrid
}

\begin{abstract}
Background: Deep surgical-site infection following thoracolumbar instrumented spinal surgery (DSITIS) is a major complication in spine surgery and its impact on long-term morbidity and mortality is yet to be determined. This article describes the characteristics and evolution of DSITIS in our center over a period of 25 years.

Methods: This single-center, retrospective cohort study included patients diagnosed with DSITIS between January 1992 and December 2016 and with a minimum follow-up after infection diagnosis of 1 year. The Infectious Diseases Society of America criteria and/or Centers for Disease Control and Prevention criteria were used to define DSITIS. Patient data (epidemiological and health status), surgical data, infection characteristics and presentation, isolated microorganisms, required surgical debridements, implant removal, and major complications linked to infection were evaluated.

Results: A total of 174 patients (106 females) were included in the analysis. Mean follow-up after infection diagnosis was 40 months (56 patients with over 5 years follow-up). Adolescent idiopathic scoliosis, adult deformity, and degenerative lumbar stenosis were the most frequent etiologies for primary surgery. Presentation of infection was considered early (0-3 months since first surgery) in 59.2\% of the cases, delayed (3-24 months) in $11.5 \%$, and late (more than 24 months) in $29.3 \%$. All patients were treated by surgical debridement. More than 1 surgical debridement was necessary in $20.7 \%$ of cases. Implants were removed in $46.6 \%$ of the patients $(72.83 \%$ in the first surgical debridement). Most frequently isolated microorganisms were Staphylococcus spp, Enterobacteriaceae, and Cutibacterium acnes. Major complications appeared in $14.3 \%$ of the patients, and over $80 \%$ of them required major surgeries to resolve those complications.

Conclusions: Late DSITIS is more frequent than previously reported. In DSITIS culprits, Staphylococcus spp, Enterobacteriaceae, and Cutibacterium acnes predominate. DSITIS produce a high rate of major complications that usually require major surgery for treatment.
\end{abstract}

Level of Evidence: 3.

Complications

Keywords: spine infection, surgical site infection, infected spinal instrumentation, early-onset infection, delayed infection, late-onset infection

\section{INTRODUCTION}

Deep surgical-site infection following thoracolumbar instrumented spinal surgery (DSITIS) is a major complication in spine surgery and its impact on long-term morbidity and mortality is yet to be determined. Infections result in prolonged hospitalization and increased costs, compromising the outcomes and life quality of patients, and may be associated with a mortality greater than $10 \% .^{1-3}$ The goals of postoperative spinal infection treatment ${ }^{3}$ are to eradicate the infection and to maintain a stable and painless spine. Treatment strategies to achieve these objectives should consider the combination of medical and surgical treatment, the triggering microorganisms, and comorbidities of the patients.

According to the relationship to the fascia, there are 2 types of infections: superficial (above the fascia) or deep (underneath the fascia). Deep infections are those that produce the highest number of therapeutic problems. ${ }^{4,5}$

Current literature regarding spinal infections is abundant but heterogeneous. ${ }^{2}$ The number of patients included tends to be low, with a short follow-up after infection diagnosis and there is no 
differentiation between superficial and deep infections and between instrumented and noninstrumented surgery.

The purpose of this study is to describe the characteristics and evolution of DSITIS in a single center over a long period of time.

\section{METHODS}

A single-center retrospective cohort study included all consecutive patients who developed DSITIS between January1992 and December 2016, with a minimum follow-up after infection diagnosis of 1year. In all cases, primary surgery was performed in our hospital. All the cases were operated by a team of 6 orthopedic surgeons, with 2 surgeons involved in each procedure. In all surgeries, at least 1 surgeon with more than 10 years of experience in spine surgery was involved. We excluded patients who presented spondylodiscitis as a primary diagnosis, superficial infections, and infections in which primary surgery was performed in another center.

The Infectious Diseases Society of America ${ }^{6}$ criteria and/or the Centers for Disease Control and Prevention ${ }^{7}$ criteria were used to define infection: purulent drainage from a superficial incision (or organ space), evidence of fistula, evidence of abscess on images or surgical revision, isolation of a microorganism in 2 or more intraoperative cultures, and surgeon clinical judgment based on preoperative and postoperative information (high temperature, swelling or redness, loosening of the instrumentation etc).

The following parameters were reviewed: epidemiological, clinical and surgical data; infection characteristics; surgical treatment; microorganisms isolated; and major complications linked to infection.

\section{Epidemiological, Clinical, and Surgical Data}

Data included age at primary surgery, gender, preoperative risk assessment (American Society of Anesthesiologists [ASA] score), body mass index (BMI), preoperative albumin levels, smoking habit, modified frailty index (mFI), ${ }^{8}$ etiological diagnosis of primary surgery, surgical approach, fused levels, surgical time, and persistent exudate of the surgical wound in primary surgery. The diagnostic value of bone scintigraphy with gallium (when available) was analyzed.

\section{Infection Characteristics}

Infection characteristics included type of presentation (serous/purulent exudate, fistula, local inflammation, fever, abscess, sepsis) and time of appearance in relation to the first surgery. Following the Zimmerli and Ochsner ${ }^{9}$ criteria, we considered early infections to be those that appeared in the first 3 months after first surgery, delayed infections between 3 and 24 months, and late infections more than 24 months thereafter.

\section{Performed Surgical Treatment}

Surgical treatment included the number of surgical debridements required by each patient and the need or not of implant removal.

Patients with early infection were treated with deep debridement and retention of implants (unless clearly loosened). A closed-suction large-bore drain was maintained until minimal output was reached (less than $30 \mathrm{~mL}$ per day). When the evolution was not satisfactory, a new surgical debridement was performed.

In delayed infections, the implant was removed or not, depending on the state of the fusion assessed by the operating surgeon.

In late infections (in which it is more likely to already have a solid fusion mass) it was usual to remove implants in the first surgical debridement.

\section{Isolated Microorganisms}

Microorganisms that grew in the cultures taken intraoperatively (at least three deep tissue samples per patient) were isolated.

Intraoperative cultures were sent for aerobic, anaerobic, fungal, and tuberculosis cultures. Cultures were held for a minimum of 14 days to assess the presence of lower-virulence microorganisms such as Cutibacterium acnes. ${ }^{10}$ This organism has a particularly long incubation period, with cultures commonly held for to 21 days as it is commonly missed if not held for sufficient time. ${ }^{11}$

\section{Major Complications Linked to Infection}

These complications were defined as adverse events after surgery that changed the expected clinical course and had consequences on the patients' health or life. ${ }^{12}$ Surgical treatment required for resolving these complications was also recorded. 


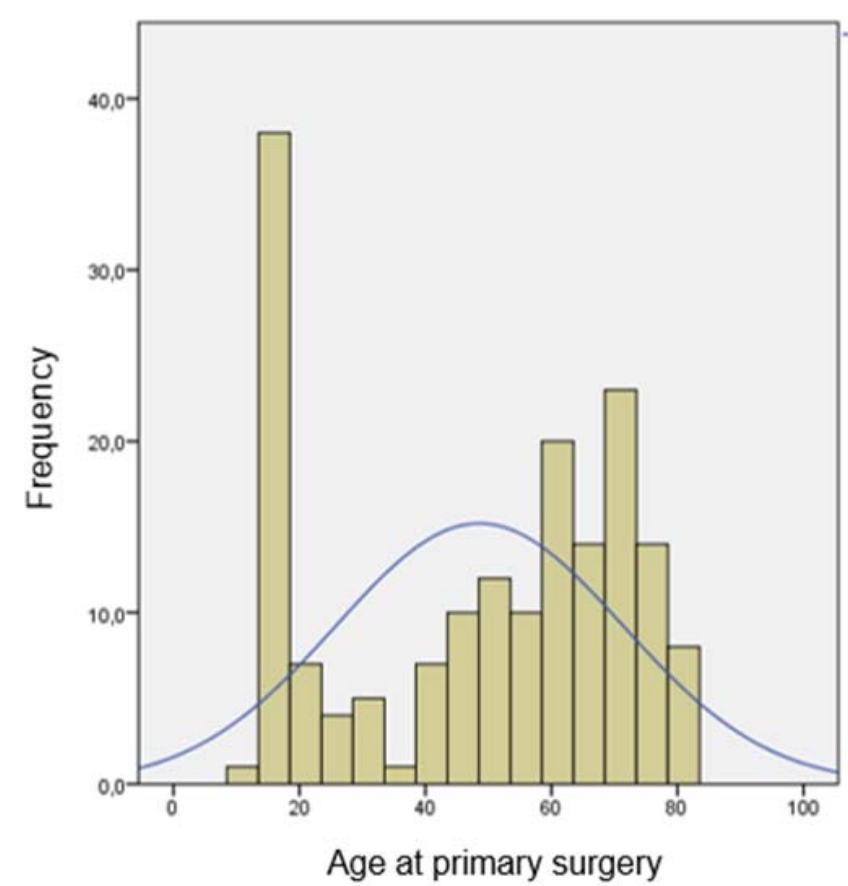

Figure. Patient distribution according to age at primary surgery.

\section{Statistical Methods and Levels of Significance}

The collected data were analyzed using IBM SPSS statistics 23 for Windows. Descriptive statistics are presented as median and interquartile range $(25 \%-75 \%)$. A $P$ value of $<.05$ was considered statistically significant.

Kolmogorov-Smirnov tests were used to test data normality. A Student $t$ test was used for normal distribution variables. A Mann-Whitney test was used for variables with nonnormal distribution.

\section{RESULTS}

\section{Epidemiological, Clinical, and Surgical Data}

A total of 174 patients (106 females and 68 males) were included in the study. The mean age at primary surgery was 48.5 years old (range: $11-82$ years). At primary surgery time, $23 \%$ of the patients were older than 70 years, but the mode of age was 16 years old (Figure).
The mean follow-up after infection diagnosis was 58.7 months (range: 12-278 months); 108 patients $(62.1 \%)$ had more than 2 years of follow-up, 93 patients $(53.5 \%)$ had more than 3 years of followup, and 56 patients $(32.1 \%)$ had more than 5 years of follow-up.

Most frequent etiologies of primary surgery were adolescent idiopathic scoliosis (35 patients), degenerative spinal stenosis (30 patients), adult deformity (17 patients), and adjacent segment disease (16 patients).

Distribution by ASA preoperative anesthetic risk was as follows, $24.7 \%$ of the patients were ASA I, 46\% ASA II, 27.6\% ASA III, and 1.7\% ASA IV. The median BMI was 27.7 (range: 11.11-45.47); $32 \%$ of the patients were overweight (BMI 25-30) and 64 patients $(36.7 \%)$ were obese $(\mathrm{BMI}>30)$. The mean modified frailty index $(\mathrm{mFI})^{8}$ was 0.08 (range: $0.00-0.45)$. The $\mathrm{mFI}$ was 0.00 in $47.7 \%$ of the cases, 0.09 in $25.3 \%, 0.18$ in $17.8 \%, 0.27$ in $6.3 \%, 0.36$ in $1.1 \%$, and 0.45 in $1.7 \%$ of them (Table 1 ).

The preoperative albumin levels (data of 107 patients were available) was $4.35 \mathrm{~g} / \mathrm{dL}$ (only 3 patients below $3 \mathrm{~g} / \mathrm{dL})$. Thirty five patients $(20 \%)$ were smokers. Clinical data of the patients included in the study are summarized in Table 2.

Eighty-nine patients $(51 \%)$ presented at least 1 clinical feature that made them prone to infection ${ }^{3}$ (Table 3).

Surgical approach was posterior in $88.5 \%$ of the cases and double in $11.5 \%$. No infection was found in any of the patients operated through a single anterior approach. Average surgical time was 5.5 hours (range: 1.60-19 hours).Average number of fused levels was 7 (range: 1-18). We performed long-segment fixations ( $>5$ levels) in $50 \%$ of the patients, and sacroiliac joint instrumentation in $58.6 \%$ of them.

Ninety-seven patients $(55.7 \%)$ that developed DSITIS presented a persistent postoperative (7-15 days) serous or hematic wound exudate during the admission of the first surgery.

Bone scintigraphy with gallium was carried out in 32 patients prior to surgical debridement and the sensitivity was $43.7 \%$.

Table 1. Modified frailty index $(\mathrm{mFl})$.

\begin{tabular}{lcccccc}
\hline & \multicolumn{5}{c}{ mFI } \\
\cline { 2 - 7 } & $\mathbf{0 . 0 0}$ & $\mathbf{0 . 0 9}$ & $\mathbf{0 . 1 8}$ & $\mathbf{0 . 2 7}$ & $\mathbf{0 . 3 6}$ & $\mathbf{0 . 4 5}$ \\
\hline No. of patients $(\%)$ & $83(47.7)$ & $44(25.3)$ & $31(17.8)$ & $11(6.3)$ & $2(1.1)$ & $3(1.7)$ \\
\hline
\end{tabular}


Table 2. Clinical data of patients with deep surgical-site infection following thoracolumbar instrumented spinal surgery (DSITIS) included in the study.

\begin{tabular}{|c|c|}
\hline Patients with DSITIS & Value \\
\hline No. of patients included & 174 \\
\hline \multicolumn{2}{|l|}{ Age, y } \\
\hline Mean & 48.59 \\
\hline Medium & $54.5(11-82)$ \\
\hline Mode & 16 \\
\hline \multicolumn{2}{|l|}{ Sex, n (\%) } \\
\hline Females & $106(60.9)$ \\
\hline Males & $68(39.1)$ \\
\hline \multicolumn{2}{|l|}{ Time elapsed since first surgery, $n(\%)$} \\
\hline$<3$ mo (early) & $103(59.2)$ \\
\hline 3-24 mo (delayed) & $19(11.5)$ \\
\hline$>24$ mo (late) & $52(29.3)$ \\
\hline \multicolumn{2}{|l|}{ Follow-up after the infection diagnosis, $\mathrm{n}(\%)$} \\
\hline$>2$ y & $108(62.5)$ \\
\hline$>3 \mathrm{y}$ & $93(53.5)$ \\
\hline$>5 \mathrm{y}$ & $56(32.1)$ \\
\hline \multicolumn{2}{|l|}{ Indication for first surgery, $\mathrm{n}(\%)$} \\
\hline Adolescent idiopathic scoliosis & $35(20.1)$ \\
\hline Degenerative spinal stenosis & $30(17.2)$ \\
\hline Adjacent-segment disease & $16(9.2)$ \\
\hline Adult deformity & $15(8.8)$ \\
\hline Tumor & $9(5.2)$ \\
\hline Fracture & $7(4)$ \\
\hline Others (degenerative disc disease, disc herniation) & $62(35.5)$ \\
\hline \multicolumn{2}{|l|}{ No. of stabilized segments } \\
\hline Mean & 7 \\
\hline Range & $1-18$ \\
\hline Long-segment fixations ( $>5$ levels), n (\%) & $87(50)$ \\
\hline \multicolumn{2}{|l|}{ Number of surgical revisions required, n $(\%)$} \\
\hline 1 surgical revision & $138(79.3)$ \\
\hline 2 surgical revisions & $25(14.4)$ \\
\hline$>2$ surgical revisions & $11(6.2)$ \\
\hline \multicolumn{2}{|l|}{ Infection type of presentation, $\mathrm{n}(\%)$} \\
\hline Serous exudate & $53(30.5)$ \\
\hline Purulent exudate & $33(19)$ \\
\hline Fistula & $44(25.3)$ \\
\hline Abscess & $8(4.6)$ \\
\hline Sepsis & $5(2.9)$ \\
\hline Local inflammation and fever, $\mathrm{n}(\%)$ & $31(17.7)$ \\
\hline
\end{tabular}

\section{Infection Characteristics}

Type of presentation of infection included the following: $49.5 \%$ presented for the first time with a serous/purulent exudate, $25.3 \%$ with a fistula, $4.6 \%$ with an abscess, $2.9 \%$ as a sepsis, and $17.7 \%$ in different ways (local inflammation, pain, fever etc).

Time of appearance according to first surgery included early infection in 103 cases $(59.2 \%)$, delayed infection in 19 cases $(11.5 \%)$ and late infection in 52 cases $(29.3 \%)$.

Table 3. Risk factors ${ }^{3}$ for acquiring postoperative infection after posterior spinal instrumentation.

\begin{tabular}{lc}
\hline Variables & No. of Patients (\%) \\
\hline Elderly (age $>70$ y) at first surgery & $34(19.54)$ \\
Body mass index $>30$ & $64(36.78)$ \\
Diabetes mellitus & $28(16.10)$ \\
Cardiovascular disease & $20(11.49)$ \\
Renal disease & $4(2.60)$ \\
Chronic pulmonary disease & $15(8.60)$ \\
Steroid use & $11(6.30)$ \\
\hline
\end{tabular}

The etiology for first surgery in late infection was adolescent idiopathic scoliosis in 26 cases $(50 \%)$, degenerative spinal stenosis in 6 cases $(11.5 \%)$, adult deformity in 5 cases $(9.6 \%)$, neuromuscular scoliosis in 4 cases $(7.7 \%)$, and others (adjacent segment disease, fracture, hyperkyphosis, etc) in 11 cases $(21.15 \%)$.

The mean time of appearance of late infection after primary surgery was 60.8 months (range: $25.1-$ 146.2 months). Out of 52 patients with late infection, 33 cases $(63.5 \%)$ presented as a fistula. Late infections appeared in younger patients (age 31.46 years versus 59.56 years, $P<.000$ ), and those with a lower $\mathrm{mFI}(0.0554$ versus $0.1022, P<.000)$ than early infections.

\section{Surgical and Medical Treatment}

One hundred and thirty-eight patients $(79.3 \%)$ required only 1 unique surgical debridement, whereas $14.4 \%$ of them required 2 debridements, and $6.2 \%$ more than 2 .

The decision for implant removal was made in patients with loosened implants or persistent infection not responsive to the first reoperation. Implant removal was necessary in 81 patients $(46.6 \%)$. In $74 \%$ of them it was carried out in the first surgical debridement, in $23.46 \%$ in the second, and in 2.54 $\%$ in the third debridement. Implant removal was performed in 20 out of 103 patients with early infection, in 15 out of 19 patients with delayed infection, and in 46 out of 52 patients with late infection. Therefore, there is a significant difference $(P<.000)$ in the percentage of implant removals performed in early, delayed, and late infections (implant removal was significantly more frequent in delayed and late infections).

In late infections (52 patients), implant removal was usually $^{13}$ carried out at the time of first debridement. Referring to early and delayed infections (122 patients), the number of days that elapsed between primary surgery and first surgical debridement was $34.7 \pm 57.2$ days in the group of patients with implant survival and $211.0 \pm 248.5$ days in the group that needed implant removal. In this group of 122 patients earlier debridement was associated with greater probability $(P<.000)$ of implant retention. Appropriately administered antibiotic prophylaxis reduces the incidence of surgical wound infection. ${ }^{14}$ Nowadays, in our hospital, the standard protocol of antibiotic prophylaxis in spine surgery is cefazolin, with $2 \mathrm{~g}$ during the anesthetic induction followed by 
3 more 1 -g doses of cefazolin every 8 hours. If surgical time is more than 4 hours or the blood loss is more than $1 \mathrm{~L}$, one more $2 \mathrm{~g}$ dose of cefazolin is administered during surgery.

The management of infection following instrumented spinal fusion was under the care of the infectious diseases team of the hospital.

Unfortunately, patients in this retrospective study were treated during different periods of time in which different treatment strategies were applied.

Currently, we treat DSITIS based on the institution's infection protocol guidelines. After surgical debridement, we start with empiric broad-spectrum antibiotic therapy (ceftazidime $2 \mathrm{~g} / 8$ hours and Vancomycin $1 \mathrm{~g} / 12$ hours) using a peripherally inserted central catheter line or central venous catheter. The infectious specialist manages the choice of definitive antibiotic therapy as soon as cultures are available. Treatment is with intravenous antibiotics in the first instance, usually for 6 weeks (although the current trend is to reduce the duration of intravenous antibiotic therapy). The switch from parenteral to oral antibiotics must be driven by the clinical outcome of the patient and occur when clinical stability is reached. The total duration is $3-6$ months.

Antibiotics are interrupted by the infectious disease specialist when patients have no clinical signs of ongoing infection and C-reactive protein is normalized.

Medical management in patients with implant removal is also varied. The cohort of our hospital had extremely varied lengths of antibiotic treatment during the period of the study.

On one hand, in patients with early infections in which implant removal and replacement of the instrumentation is performed, we tend to be more aggressive with longer periods of antibiotic treatment (usually a total duration of more than 6 months).

On the other hand, in patients with delayed and late infections in which implant removal is performed without implant replacement (because they have a solid mass of fusion), the optimal duration of therapy is yet to be determined. ${ }^{13}$

In our hospital, we tend to treat these patients using a less aggressive approach than in patients with retained hardware. These patients usually complete a course of intravenous antibiotics from 2 to 4 weeks and a total duration of treatment of $2-4$ months depending on clinical and analytic results.
Wound and soft tissue closure treatment strategies have changed over the past years; therefore, patients in this retrospective cohort were treated with different techniques over the period of the study.

Adequate soft tissue coverage of the postoperative spine is a requirement for successful patient outcome. Currently, in our hospital, we have a standard protocol to close the wound. In the first surgical debridement (which is the only one in most cases), we perform primary closure of the wound (always if the tissue has no necrosis and is clean at the end of the debridement).

If after the debridement the tissue looks questionable or in the cases of required subsequent debridements to obtain a clean wound for closure, we request the assessment of reconstructive surgery service during the procedure. Based on the reconstructive surgeon's criteria and individualizing in each patient (depending on the skin and soft tissue status) they perform different procedures:

- Vacuum-assisted wound closure system is an effective adjunct increasingly used in closing complex deep spinal wounds with exposed instrumentation. It exposes the wound bed to negative pressure, resulting in removal of edema fluid, improvement of blood supply, and stimulation of cellular proliferation of reparative granulation tissue. ${ }^{15}$ Usually, patients need more than 1 trip to the operating room to repeat the debridement and the vacuum-assisted closure system until the wound is clean and ready to close.

- In complex wounds with contracted scars that are difficult to close, reconstructive surgeons perform Z-plasty techniques of wound closure. This technique seems to be a good alternative to conventional midline incision closure with the potential to reduce the rate of delayed wound healing and wound infection.

- In patients with acquired midline back defects and loss of soft tissue coverage resulting from multiple surgeries, reconstructive surgeons perform muscle-flap wound closures. This procedure facilitates the establishment of a soft tissue envelope to achieve short- and longterm wound healing. ${ }^{16}$

In our experience, we have noticed that patients with spine tumors that were previously treated with 
Table 4. Major complications linked to infection and its treatment.

\begin{tabular}{ll}
\hline No. of Cases of Each Complication & \multicolumn{1}{c}{ Treatment } \\
\hline Pseudoarthrosis with loss of correction: 10 & 9 double approaches \\
Pseudoarthrosis with loss of correction: 2 & 5 osteotomies \\
Pseudoarthrosis with loss of correction: 2 & Revision surgery by posterior approach \\
& 1 double approach with osteotomies (exitus) \\
& 1 reinstrumentation; reinfection \\
Implant dislodgement with loss of correction: 1 & Multiple debridements required \\
Pressure sores: 3 & Partial implant removal \\
Adjacent-disc disease: 4 & Reconstructive surgery \\
Deaths: 3 & Spinal fusion extension through a costotransversectomy approach \\
\end{tabular}

radiotherapy are at high risk of wound healing problems. In order to avoid these problems, we use preventatively in the first surgery a negativepressure wound therapy dressing with good results.

\section{Isolated Microorganisms}

In almost half of early-infection cases Staphylococcus spp were isolated; Enterobacteriaceae (Escherichia coli, Proteus spp, and Enterococcus spp) were isolated in nearly the other half.

Cutibacterium acnes and coagulase-negative Staphylococcus were the most frequently isolated microorganisms in delayed and late infections. In $14 \%$ of the patients, analyzed samples were culturenegative despite clinical signs of infection. In samples taken in the first surgical debridement, 3 or more microorganisms were isolated in 24 out of 174 cases $(13.79 \%)$.

When a second surgical debridement was necessary ( 36 cases), cultures were negative in 5 cases, and more frequently isolated microorganisms were Enterobacteriaceae (almost one-third of cases) and coagulase-negative Staphylococcus. In $50 \%$ of these patients that were submitted to a second surgical revision, new microorganisms that were not present in the first cultures were isolated.

\section{Major Complications Linked to Infection}

During follow-up, major complications linked to infection appeared in 25 patients $(14.3 \%)$. The most frequent complication was pseudoarthrosis with loss of correction.

Most of those complications (88\%) required major surgeries (osteotomies, double approaches etc) for resolution. There were 3 deaths caused by infection (all deaths were caused by sepsis). Major complications appeared in 17 patients $(68 \%)$ with early infection, in 4 patients $(16 \%)$ with delayed infection, and in 4 patients $(16 \%)$ with late infection. Table 4 shows these complications and surgeries performed in order to treat them.

\section{DISCUSSION}

The incidence of infection in spinal surgery and the risk factors for its development are profusely described in the literature and are not the purpose of this study. Out of 3000 thoracolumbar fusions performed over 25 years, we report 174 occurrences of DSITIS. However, an incidence analysis was not the purpose of this study. Due to the long follow-up, some patients were lost in follow-up; therefore, incidence analysis in our series could underestimate the true occurrence of DSITIS. Incidence and risk factors vary according to the series and the infection definition. $^{17}$

From a descriptive standpoint, we observed that $36.7 \%$ of the infected patients were obese, $20 \%$ smokers, and $16 \%$ diabetics. In half of the patients, long instrumentation ( $>5$ levels) was performed with an average surgical time above 5 hours. In a systematic review ${ }^{2}$ that included more than 2000 patients, the authors identified prolonged surgical time, diabetes, smoking habit, previous infection, surgical aggressiveness, and a high BMI as risk factors with strong evidence for acquiring postoperative infection. The risk of infection seems to be proportional to the increase of BMI. ${ }^{18,19}$

Prevention of surgical-site infection in spine surgery includes a wide variety of measures directed to reduce all the changeable risk factors as diabetes mellitus, obesity, or smoking habit.

Diabetes mellitus patients whose blood glucose level are poorly controlled before surgery are at high risk for develop surgical-site infection. ${ }^{20}$ In order to prevent this situation, we recommend lowering the hemoglobin A1c of every diabetic patient to $<7 \%$ before performing surgery.

Obesity (BMI $>30 \mathrm{~kg} / \mathrm{m}^{2}$ ) has been identified as a significant risk factor for surgical-site infection. ${ }^{21}$ 
Therefore, obese patients are advised to lose weight before surgery.

Smoking is probably the most important modifiable risk factor for surgical-site infection following implant insertion. ${ }^{22}$ As a result, we must insist patients to stop smoking preoperatively or consider smoking cessation programs in patients undergoing surgery with implant materials. ${ }^{23}$

A higher surgical risk $^{2,8,19}$ of the patients assessed by ASA and $\mathrm{mFI}$ has been associated with a higher rate of complications and infection. In our series, both ASA and mFI values were low. This could be explained due to the fact that the vast majority of our surgeries were elective cases and were not performed in patients with poor health status.

Age over 70 is considered to be a risk factor for infection. $^{2,3}$ In our study, almost $20 \%$ of the patients were over 70 at the time of primary surgery. However, it is important to highlight that mode age in our series was 16 years. This fact may be related to the high percentage of adolescent idiopathic scoliosis $(20 \%$ within the whole series).

Almost $30 \%$ of our infections were classified as "late" infections and this figure is much higher than previously reported. ${ }^{3,13,24}$ Late infection typically presents after a long follow-up in adolescents and adult patients who underwent surgery for spinal deformity. In these patients, long instrumented constructs provide a large surface on which microorganisms can adhere. The clinical course of late infection is insidious and it is usually diagnosed after the occurrence of a fistula. In late infections skin microorganisms predominate in cultures. We believe that it is important to be alert about this type of late infection in deformity surgery and specifically informing about its possible appearance to patients who require long spinal instrumentation. We recommend long follow-ups for these patients in order to detect these infections early.

The remaining infections (early and delayed) are associated with older age and worse preoperative general health status in patients.

More than half of our patients presented persistent surgical wound exudate in the postoperative period of the primary surgery. The role of this kind of persistent exudate is not clearly defined as a possible risk factor for infection, although in some series $^{2}$ it is indicated as such.

The most frequent presentation of infection in this series was serous or purulent exudate. One of the limitations of this retrospective study is that we were not allowed to exhaustively study the presence of pain as a symptom of infection, but in our clinical experience, as previously reported, ${ }^{25}$ persistent and continuous pain in the infected region that does not yield to analgesics is a very frequent symptom. In patients with late infections, pain radically disappears when the implants are removed. Insidious pain should lead to clinical suspicion of a possible underlying infection.

In our series, the sensitivity of the bone scintigraphy with gallium for the infection diagnosis was very low. There are articles ${ }^{25}$ that report a $90 \%$ accuracy of bone scintigraphy in diagnosis of bone infection, but that literature mainly refers to primary spondylodiscitis diagnosis and not to the diagnosis of infection in instrumented spinal surgery. It is likely that positron emission tomography and computed tomography with fluorodeoxyglucose F-18 could be of more value than bone scintigraphy for the instrumented spinal surgery infection diagnosis and selective treatment. ${ }^{26}$

We applied the Zimmerli and Ochsner ${ }^{9}$ classification for early, delayed, late infections because of its recognition in prosthetic surgery. In spinal fusion surgery, after 2 years following surgery, usually a solid fusion mass is achieved. Once the arthrodesis is well consolidated, implant removal can be performed without risk of instability. However, it is known that the graft blocks are dynamic and that, despite seeing a good posterior intraoperative graft when performing implant removal, the patient may lose correction ${ }^{27}$ after removing the instrumentation. In infections that are not late $(<24$ months after surgery), we believe that surgical debridement is recommended as early as possible, since we have observed, as previously reported, ${ }^{28}$ that earlier debridement helps implant retention, minimizing the risk of instability. The decision to carry out implant removal and the replacement of instrumentation if it is considered necessary must be individualized in each patient, depending on the arthrodesis mass, time of evolution, the type of microorganism, biofilms, sensitivity to antibiotic treatment, detritus found at surgery time, and the surgeon's experience.

Most frequently isolated microorganisms were Staphylococcus spp, Enterobacteriaceae, and Cutibacterium acnes. Skin microorganisms (such as coagulase-negative Staphylococcus and Cutibacterium acnes) were frequently isolated, especially in delayed and late infections. Previous studies identi- 
fied Staphylococcus aureus as the most frequently isolated pathogen, ${ }^{2,3,12}$ and there is recent evidence $^{24}$ supporting our findings.

In patients who needed a second surgical revision (given bad evolution), we have observed that, in half of the cases, microorganisms different from those grown in the first surgical revision were isolated (superinfection). As far as we know, this finding has never been previously reported in the literature. From our point of view, in patients with torpid evolution after first surgical debridement, it is very important to have high clinical suspicion of superinfection and it is also important to hold the cultures a minimum of 14-21 days in order to detect the presence of lower-virulence microorganisms.

Deep infection is associated $d^{3,5,29,30}$ with a greater number of complications, a large percentage of pseudoarthrosis, a greater number of revision surgeries, poorer quality of life, and a mortality that can exceed $10 \%$. Although pseudoarthrosis appeared to be the most frequent complication of DSITIS in our series we must bear in mind that infection may develop in a patient with an established pseudoarthrosis and may or may not be a related finding. This piece of data agrees with our clinical experience. The most severe complications that require more aggressive surgeries occur mostly in adults, but not exclusively.

Since this study does not include surgeries performed by unsupervised junior surgeons, the effect of surgeon experience on DSITIS could not be adequately assessed. Although this review presents the limitations of a retrospective study, and does not refer to the antibiotic treatment performed, it has a prolonged mean follow-up after infection diagnosis, reveals the high percentage of late infection, and warns of the complications linked to infection and the need of very aggressive revision surgeries. We are preparing another paper analyzing more exhaustively the microorganisms that cause the infection, but in this review we point to the importance of skin microorganisms, and that in cases of poor evolution after surgical debridement and correct antibiotic treatment, superinfection may exist.

\section{CONCLUSIONS}

The number of late infections (2 years after primary surgery) in instrumented spinal surgery represents a high percentage of total infections. They present mainly in patients operated for deformity with long instrumentations and long follow-up. Patients should be informed about this possible complication in the long run.

In early and delayed infections, earlier debridement is associated with greater probability of implant retention. Bone scintigraphy with gallium showed low sensitivity for infection diagnosis.

Considering whole series, Staphylococcus spp, Enterobacteriaceae, and Cutibacterium acnes are the most frequently isolated microorganisms.

DSITIS entails a high percentage of complications, and almost $90 \%$ of those complications require major surgeries to resolve.

\section{REFERENCES}

1. Casper DS, Zmistowski B, Hollern DA, et al. The effect of postoperative spinal infections on patient mortality. Spine. 2018; 32(3):223-227.

2. Xing D, Ma J-X, Ma X-L, et al. A methodological, systematic review of evidence-based independent risk factors for surgical site infections after spinal surgery. Eur Spine J. 2013;22(3):605-615.

3. Chen $\mathrm{SH}$, Lee $\mathrm{CH}$, Huang $\mathrm{KC}$, Hsieh PH, Tsai SY. Postoperative wound infection after posterior spinal instrumentation. Analysis of long-term treatment outcomes. Eur Spine J. 2015;24(3):561-570.

4. Pull ter Gunne AF, Mohamed AS, Skolasky RL, Van Laarhoven CJ, Cohen DB. The presentation, incidence, etiology and treatment of surgical site infections after spinal surgery. Spine. 2010;35(13):1323-1328

5. Mok JM, Guillaume TJ, Talu U, et al. Clinical outcome of deep wound infection after instrumented posterior spinal fusion: a matched cohort analysis. Spine. 2009;34(6):578-583.

6. Osmon DR, Berbari EF, Berendt AR, et al. Diagnosis and management of prosthetiLovec joint infection: clinical practice guidelines by the Infectious Diseases Society of America. Clin infect Dis. 2013;56(1):e1-e25.

7. Centers for Disease Control and Prevention. Surgical site infection (SSI) event January 2016. http://www.cdc.gov/ngsn/ acute-care-hospital/ssi/. Accessed May 5, 2016.

8. Leven DM, Lee NJ, Kothari P, et al. Frailty index is a significant predictor of complications and mortality after surgery for adult spinal deformity. Spine. 2016;41(23):E1394E1401.

9. Zimmerli W, Ochsner PE. Management of infection associated with prosthetic joints. Infection. 2003;31(2):99-108.

10. Steinhaus M, Salzmann SN, Lovecchio F, et al. Risk factors for positive cultures in presumed aseptic revision spine surgery. Spine. 2019;44(3):177-184.

11. Brook I, Frazier EH. Infections caused by Propionibacterium species. Rev Infect Dis. 1991;13(5):819-822.

12. Lebude B, Yadla S, Albert T, et al. Defining "complications" in spine surgery: neurosurgery and orthopedic spine surgeons' survey. J Spinal Disord Tech. 2010;23(8):493-500.

13. Garg S, LaGreca J, Hotchkiss M, Erickson M. Management of late $(>1 \mathrm{y})$ deep infection after spinal fusion: a retrospective cohort study. J Pediatr Orthop. 2015;35(3):266270 .

14. Setiawan B. The role of prophylactic antibiotics in 
preventing perioperative infection. Acta Med Indones. 2011;43(4):262-266.

15. Mehbod AA, Ogilvie JW, Pinto MR, et al. Postoperative deep wound infections in adults after spinal fusion: management with vacuum-assisted wound closure. J Spinal Disord Tech. 2005;18(1):14-17.

16. Klink BK, Thurman RT, Wittpenn GP, Lauerman WC, Cain JE. Muscle flap closure for salvage of complex back wounds. Spine (Phila Pa 1976). 1994;19(13):1467-1470.

17. Nota SP, Braun Y, Ring D, Schwab JH. Incidence of surgical site infection after spine surgery: what is the impact of the definition of infection? Clin Orthop Relat Res. 2015;473(5):1612-1619.

18. Pull ter Gunne AF, Cohen DB. Incidence, prevalence, and analysis of risk factors for surgical site infection following adult spinal surgery. Spine. 2009;34(13):1422-1428.

19. Puffer RC, Murphy M, Maloney P, et al. Increased total anesthetic time leads to higher rates of surgical site infections in spinal fusions. Spine. 2017;42(11):E687-E690.

20. Hikata T, Iwanami A, Hosogane N, et al. High preoperative hemoglobin A1c is a risk factor for surgical site infection after posterior thoracic and lumbar spinal instrumentation surgery. J Orthop Sci. 2014;19(2):223-228.

21. Pull ter Gunne AF, Hosman AJF, Cohen DB, et al. A methodological systematic review on surgical site infections following spinal surgery: part 1: risk factors. Spine (Phila $\mathrm{Pa}$ 1976). 2012;37(24):2017-2033.

22. Ferry T, Valour F, Lustig S, et al. The challenge of infection prevention in spine surgery: an update. Eur J Orthop Surg Traumatol. 2013;23(suppl 1):S15-S19.

23. Durand F, Berthelot P, Cazorla C, Farizon F, Lucht F. Smoking is a risk factor of organ/space surgical site infection in orthopaedic surgery with implant materials. Int Orthop. 2013;37(4):723-727.

24. Dapunt U, Bürkle C, Günther F, et al. Surgical site infections following instrumented stabilization of the spine. Ther Clin Risk Manag. 2017;13:1239-1245.

25. Chahoud J, Kanafani Z, Kanj SS. Surgical site infections following spine surgery: eliminating the controversies in the diagnosis. Front Med (Lausanne). 2014;1:7.

26. Wang Y, Cheung JP, Cheung KM. Use of PET/CT in the early diagnosis of implant related wound infection and avoidance of wound debridement. Eur Spine J. 2016;25(suppl 1):38-43.

27. Muschik M, Lück W, Schlenzka D. Implant removal for late-developing infection after instrumented posterior spinal fusion for scoliosis: reinstrumentation reduces loss of correction. A retrospective analysis of 45 cases. Eur Spine J. 2004;13(7):645-651.

28. Tsubouchi N, Fujibayashi S, Otsuki B, et al. Risk factors for implant removal after spinal surgical site infection. Eur Spine J. 2018;27(10):2481-2490.

29. Wang TY, Back AG, Hompe E, Wall K, Gottfried ON. Impact of surgical site infection and surgical debridement on lumbar arthrodesis: a single-institution analysis of incidence and risk factors. J Clin Neurosci. 2017;39:164-169.

30. Haddad S, Núñez-Pereira S, Pigrau C, et al. The impact of deep surgical site infection on surgical outcomes after posterior adult spinal deformity surgery: a matched control study. Eur Spine J. 2018; 27(10):2518-2528.

Disclosures and COI: The authors declare no conflict of interest.

Corresponding Author: Borja de la Hera, MD, Department of Orthopedic Surgery, Getafe University Hospital, Carretera de Toledo km 12,500, Madrid 28905, Getafe, Madrid, Spain. Phone: (+34) 616363816; (+34) 916839360; Fax: (+34) 9168397 48; Email: borjadelahera@ hotmail. com.

Published 26 February 2021

This manuscript is generously published free of charge by ISASS, the International Society for the Advancement of Spine Surgery. Copyright $\odot 2021$ ISASS. To see more or order reprints or permissions, see http://ijssurgery.com. 